Box 1. Proposed DSM-5 Suicide Assessment Dimension

Each of the 7 items shown below are coded as present (1) or absent (0); ratings are summed to yield an indication of level of concern about potential suicidal behaviour (0-lowest concern, 1-2 some concern, 3-4 increased concern, 5-7 high concern).

1. Any history of suicide attempt.

2. Long-standing tendency to lose temper or become aggressive with little provocation.

3. Living alone, chronic severe pain, or recent (within 3 months) significant loss.

4. Recent psychiatric admission/discharge or first diagnosis of MDD, bipolar disorder or schizophrenia.

5. Recent increase in alcohol abuse or worsening of depressive symptoms.

6. Current (within last week) preoccupation with, or plans for suicide.

7. Current psychomotor agitation, marked anxiety or prominent feelings of hopelessness.

among suicide decedents in that study. Further, low openness to experience has been associated with death by suicide in later life (Duberstein et al., 1994). We therefore propose that Item 2 could include also rigid personality style.

Item 3 (living alone, chronic severe pain, or recent (within 3 months) significant loss) begs comment from a late-life perspective. Many seniors live alone, but few die by suicide. Lack of social support (Harrison et al., 2010) would constitute a more compelling signal of increased risk for suicidal behavior in this age group. Further, the three-month specifier for significant loss seems short. Complicated grief, which is diagnosed after six months or more, has been shown to be related to suicidality (Szanto et al., 2006). Risk may be systematically underrated in seniors as Item 3 factors are more likely to co-exist and interact in later life.

Older persons with severe physical illness/ functional disability are more likely to commit suicide than their healthier peers (Waern et al.,

doi:10.1017/\$1041610210002000

\section{The safe application of physical interventions in aggressive older adults: considerations from the physiotherapy profession}

In certain older adult subpopulations, e.g. those with dementia or cognitive impairment, aggression would appear to be commonplace, particularly in inpatient settings (Winstanley and Whittington, 2004; Stewart et al., 2008). Healthcare profession-
2002). Serious health problems can lead to loss of autonomy; individuals may feel that they are a burden on others. A separate rating for "serious physical health issues" (chronic severe pain/severe physical illness/disability) might enhance the utility of the Suicide Assessment Dimension for the elderly, a vulnerable and fast-growing age group.

Note: The case is a composite based on a psychological autopsy study of suicides that occurred in late life (Waern et al., 2002).

\section{References}

Duberstein, P. R., Conwell, Y. and Caine, E. D. (1994). Age differences in the personality characteristics of suicide completers: preliminary findings from a psychological autopsy study. Psychiatry, 57, 213-224.

Harrison, K. E. et al. (2010). Alone? Perceived social support and chronic interpersonal difficulties in suicidal elders. International Psychogeriatrics, 22, 445-454.

McGirr, A., Renaud, J., Bureau, A., Seguin, M., Lesage, A. and Turecki, G. (2008). Impulsive-aggressive behaviours and completed suicide across the life cycle: a predisposition for younger age of suicide. Psychological Medicine, 38, 407-417.

Szanto, K. et al. (2006). Indirect self-destructive behavior and overt suicidality in patients with complicated grief. Fournal of Clinical Psychiatry, 67, 233-239.

Waern, M., Rubenowitz, E., Runeson, B., Skoog, I., Wilhelmson, K. and Allebeck, P. (2002). Burden of illness and suicide in elderly people: case-control study. BMF, 324, 1350-1355.
M. WAERN, ${ }^{1}$ A. Y. DOMBROVSKI ${ }^{2}$ AND
K. SZANTO ${ }^{2}$
${ }^{1}$ Section of Psychiatry, Sahlgrenska Academy at the University of Gothenburg, Gothenburg, Sweden
${ }^{2}$ Western Psychiatric Institute and Clinic, Dept. of
Psychiatry, University of Pittsburgh School of
Medicine, Pittsburgh, PA, U.S.A.
Email: margda.waern@neuro.gu.se

als have a spectrum of different techniques they may employ to avoid and manage aggression (National Institute of Clinical Excellence, 2005). Aggression may manifest itself in many forms, but physical assault is particularly troublesome as this may have many deleterious effects on the victim's health. The displays of such behavior increase the likelihood that more intrusive measures, such as physical intervention and/or administration of psychotropic medication, are used by the responding healthcare professionals (NICE, 2005). 
One method of dealing with physical assault is physical intervention, or physical restraint. Physical intervention involves a team of trained staff, usually two or more, applying physical techniques to restrict or move the aggressive patient so that safety is restored in the clinical setting (NICE, 2005). It is imperative that healthcare staff use physical intervention as an absolute last resort, when all other less intrusive interventions - for example, deescalation - have failed (NICE, 2005). Clearly there is a risk that the application of physical intervention may cause pain or injury to the assailant, something which needs to be avoided if at all possible (NICE, 2005). It would appear that the majority of physical intervention training courses teach generic techniques, which largely consider the needs of adults who have no presenting physical ailments. Despite this, injury rates among working age adults from the application of physical intervention have been reported to be as high as almost one in every five incidents of physical intervention. Thus, the application of physical intervention in older adults may cause injury to a considerable number of recipients when one considers the often more complex physical presentation of this population (Stubbs et al., 2008). For this reason, we believe that physical intervention techniques should be adapted appropriately to consider the complex needs of this population. Physiotherapists may provide screening assessments to identify any pre-existing physical ailments and work in conjunction with a specialist physical intervention tutor to advise the clinical team on appropriate adaptive techniques to reduce the risk of the application of the techniques causing pain and/ or injury (Stubbs et al., 2008). Stubbs and colleagues (2008) reported that this approach resulted in considerably lower levels of injury to patients $(2 \%)$, which is considerably lower than injury rates reported in working age adults.

We believe that physiotherapists have an integral role in helping to reduce the risk of injury to aggressive older adults from the application of physical intervention. In a recent national survey, Stubbs (2010) revealed that a quarter of physiotherapists working in mental health had concerns that the application of physical intervention would have a deleterious effect on the recipient's health. In this national survey, (response rate 65\%), the mental health physiotherapists were asked to make recommendations for safer application of physical intervention in the psychiatric population within which they were working. Some of the physiotherapists $(n=15)$ working in older adult psychiatry made recommendations about the safe application of physical intervention in this patient group. Eight of them stated that wrist flexion to induce pain compliance should not be used, but that passive holds at the wrist and above the wrist joint should be implemented instead. We concur that this should be adopted, since the risk of causing a colles fracture, for instance, is elevated in this population.

Seven physiotherapists expressed concerns about the application of neck flexion and advised that this should be avoided wherever practicable; something which we believe is a better solution. Ten physiotherapists expressed concerns about the risk of shoulder subluxation from the application of the techniques. Indeed, three physiotherapists reported that they were actively treating an older adult following an injury of this nature. Clearly, with the changes in muscle tone and sensation, the shoulder is vulnerable to injuries of this nature. Previous research in this population (Stubbs et al., 2008) has demonstrated that the upper limb is injured most frequently following the application of physical intervention. This has also been demonstrated in adults with acquired brain injury who may also experience similar changes in muscle tone and sensation. For this reason, we recommend that end of range movements should not be used and that internal and external rotation should be avoided wherever possible, since such movements are known to increase the likelihood of causing an individual pain and/or subluxation.

Finally, eleven physiotherapists strongly recommended that "going to the floor" should be avoided at all costs. We agree with this, since previous research by the first author has demonstrated that older adult psychiatric inpatients have a high incidence of osteoporosis and osteopenia. The "takedown" to the floor may result in fracture, with the hip being particularly vulnerable. Fractures to the hip are associated with much morbidity and mortality and indeed many die following such trauma. For this reason, we agree that going to the floor should be avoided at all costs.

In summary, older adult psychiatric inpatients may display severe aggressive behavior and physical intervention should be used as a last resort to manage this behavior (Stewart et al., 2008). The application of physical intervention is complex in this population and in this letter we make some provisional recommendations to reduce the risk of causing pain and/ or injury to the aggressive older adult.

\section{References}

National Institute of Clinical Excellence (2005). Violence: The Short-Term Management of Disturbed/ Violent Behavior in 
In-Patient Psychiatric Settings and Emergency Departments. London: National Institute of Clinical Excellence.

Stewart, I., Knight, C. and Johnson, C. (2008). Just how challenging can older people be? Part 2: Making the case for specialist services for risky and aggressive behavior. PSIGE Nerwsletter, 103, 66-74.

Stubbs, B. (2010). Physiotherapist involvement and views on the application of physical intervention to manage aggression: data from a national survey. Fournal of Psychiatric and Mental Health Nursing, 17, 754-756.

Stubbs, B., Yorston, G. and Knight, C. (2008) Physical intervention to manage aggression in older adults: how often is it employed? International Psychogeriatrics, 20, 855-857.

doi:10.1017/S1041610210001912

\section{Rehabilitation for dementia using enjoyable video-sports games}

The aging of society inevitably leads to an increase in the numbers of elderly with dementia who reside in nursing homes, and delaying disease progression of residents with dementia has become a big concern. Rehabilitation that focuses directly on training cognitive function (e.g. memory training) reveals what patients are unable to do. Realization of their cognitive deficits can devastate their selfconfidence and lead to anxiety, depression and the lowering of self-esteem (Small et al., 1997). We propose rehabilitation that encourages patients' motivation for self-improvement through social interaction based on five principles as follows: (1) the activities should be enjoyable and comfortable for patients, (2) therapists should praise the patients naturally to motivate them, (3) the activities should be associated with empathetic two-way communication to make patients feel valued and safe, (4) therapists should encourage the patients to play "social roles" to restore self-worth, and (5) error-less learning based on brain-activating rehabilitation (BAR; Yamaguchi et al., in press) should be adopted wherever possible. It is suggested that the positive feelings activate those areas of the brain related to reward, which plays a critical role in motivation (Berridge et al., 2003), and it is a typical social reward to be praised and appreciated in public.

Based on BAR, we tried to improve residents' cognitive function indirectly by enhancing motivation using enjoyable video-sports games in a group setting. We conducted interventions with nine elderly people with mild to moderate dementia $(88.9 \pm 4.9$ years of age, mean $\pm S D$; three males and six females: one with Parkinson's disease dementia, one with vascular dementia, and seven
Winstanley, S. and Whittington, R. (2004). Aggressive encounters between patient and healthcare staff: the context and assailants levels of cognitive processing. Aggressive Behaviour, 30, 534-543.

\author{
BRENDON STUbBs ${ }^{1}$ AND LeE Hollins ${ }^{2}$ \\ ${ }^{1}$ Clinical Specialist Mental Health Research \\ Physiotherapist, Northampton, U.K. \\ Email: brendonstubbs@hotmail.com \\ ${ }^{2}$ Physiotherapist and Physical Intervention Tutor, \\ London, U.K.
}

with Alzheimer's disease (AD)) residing in a nursing home. All were in the stable phase of dementia, and had been admitted to the nursing home at least three months previously. None of the participants was medicated with donepezil hydrochloride.

We used video sports-games specifically devised for rehabilitation (Hot-plus, SSD Co. Ltd, Shiga Japan). These utilize psychomotor skills, such as hand-eye coordination, require timing, and necessitate fine three-dimensional control of the limbs in space. There were essentially two types of games: those working the upper limbs, and those the lower limbs. An example of the games for upper limbs required a player to grab coins which appeared to be coming out of the TV screen. The players wore bands on their hands equipped with sensors, and when the timing and direction were accurate, they scored points. Games for the lower limbs included those which required the players to move their legs to music. Two Japanese drums were shown on the TV screen, and two balls fell slowly from the top of the TV screen. A player was required to tap his/her feet on a mat equipped with sensors, synchronizing with the balls bouncing on the drums. $\mathrm{He} / \mathrm{she}$ scored when the timing was accurate. These interventions were conducted once a week for ten weeks.

General cognitive function was measured using Hasegawa's Dementia Scale-revised (HDS-R), which is similar and well-correlated with the Minimental State Examination. The visuospatial and constructive function was measured using Kohs block-design tests (Kohs). Behavioral changes were evaluated using the Multidimensional Observation Scale for Elderly Subjects (MOSES), with the subitems of self-care, disorientation, depression, irritability, and withdrawal. No residents had previously experienced playing the video-sports games and so to help the residents enjoy the games, the caregivers (aged $40.8 \pm 12.9$ years, 7 males and 